\title{
Community composition of rhizosphere fungi as affected by Funneliformis mosseae in soybean continuous cropping soil during seedling period
}

\author{
Weiguang Jie ${ }^{1,2}$, Jixiang Lin ${ }^{1}$, Na Guo ${ }^{1,2}$, Baiyan Cai ${ }^{3}$, and Xiufeng Yan ${ }^{1 *}$ \\ ${ }^{1}$ Northeast Forestry University, Alkali Soil Natural Environmental Science Center, Ministry of Education, Harbin 150040, China. \\ "Corresponding author (xfyan@nefu.edu.cn). \\ ${ }^{2}$ Heilongjiang East University, Department of Food and Environment Engineering, Harbin 150066, China. \\ ${ }^{3}$ Heilongjiang University, College of Life Sciences, Harbin 150080, China.
}

Received: 16 December 2018; Accepted: 2 April 2019; doi:10.4067/S0718-58392019000300356

\begin{abstract}
Arbuscular mycorrhizal (AM) fungi can enhance plant resistance particularly against soil-borne pathogenic fungi. However, little is known about the effects of Funneliformis mosseae on the community composition of rhizosphere fungi in soybean (Glycine $\max$ [L.] Merr.) continuous cropping soil. Here, the disease index of soybean root rot was analyzed, and high throughput sequencing technology was applied to investigate whether $F$. mosseae could change the composition of fungal communities in the rhizosphere of continuous cropping soybean during seedling period. The results indicated that the disease index of soybean root rot decreased significantly after inoculation of $F$. mosseae. The root rot disease index was also affected by the increasing of continuous cropping regimes. Furthermore, the relative abundance of fungal community in soybean rhizosphere soil and root samples was influenced after inoculation. Ascomycota was the dominant phylum in most samples. Basidiomycota was the second dominant phylum in all the soil samples, but Olpidiomycota was the second phylum in most root samples. At the genus level, both inoculation and continuous cropping regimes had significant effects on the dominant genus and their relative abundances in all the samples. The relative abundance of some plant pathogenic fungi such as Fusarium in the inoculated root samples was lower than those in the non-inoculated root samples in the same continuous cropping regime. The results can provide new insights into the interactive effects of AM fungi and rhizosphere fungi, and also provide theoretical evidence on biological solutions to alleviate the obstacles of soybean continuous cropping.
\end{abstract}

Key words: Continuous cropping, fungal communities, Glycine max, Funneliformis mosseae, high throughput sequencing, soybean.

\section{INTRODUCTION}

Soybean (Glycine max [L.] Merr.) is a main grain and oilseed crop in the word. The cultivation areas of soybean are mainly distributed in America, Brazil, Argentina and China (Gawade et al., 2017). Soybean is a kind of crop with continuous cropping obstacles. Continuous cropping can lead to a decline in crop yield and quality (Huang et al., 2013). Lack of soil nutrients, changes of soil physical structure, autotoxicity of allelochemicals from root exudates, and enrichment of soilborne diseases are the possible causes of continuous cropping obstacles (Liu et al., 2017). Root rot is one of the serious soybean diseases in China (Jeon et al., 2013).

Rhizosphere microorganisms are mainly composed of fungi, bacteria and actinomycetes (Deng et al., 2018). The composition of rhizosphere microbial community is influenced by soil types and plant species (Berg and Smalla, 2009). Especially in soybean, the composition of rhizosphere microbial communities is different from other plants, because it 
has a strong ability to form symbiotic relationships with many microorganisms (Sugiyama et al., 2014). Furthermore, the structure of the soil microbial community can also reflect soil ecological environment (Costa et al., 2006). Many studies have shown that the structure, abundance and diversity of rhizosphere microbial communities are essential for maintaining soil quality (Garbeva et al., 2004; Kong et al., 2011).

Arbuscular mycorrhizal (AM) fungi are oligotrophic microorganisms, belonging to the phylum Zygomycota (Spatafora et al., 2016), it can maintain soil fertility and high-quality crop production (Battini et al., 2016). Arbuscular mycorrhizal fungi can contribute to soil aggregation and stability by producing glomalin (Wright and Upadhyaya, 1998); AM fungi can improve rhizosphere nutrition, enhance plant resistance to various pathogens and promote plant growth (Wang et al., 2018). The inoculation effects of AM fungi in maize (Dhawi et al., 2015), wheat (Zhu et al., 2017) and soybean (Spagnoletti et al., 2017) have been proved.

To clarify the changes in the community composition of rhizosphere fungi in soybean resulting from different continuous cropping regimes, it is very important to choose suitable management measures to improve the function of soil ecosystem. So far, there have been many researches detecting the community composition of rhizosphere microorganisms from different plants under continuous cropping systems, including cotton, tomato and notoginseng (Li et al., 2014; Luan et al., 2015; Wu et al., 2016). However, little is known about the effects of Funneliformis mosseae on the community composition of rhizosphere fungi in soybean continuous cropping soil during the seedling period.

Therefore, the objectives of this study were to explore (1) whether inoculation of $F$. mosseae could exert a positive effect on the root rot incidence of soybean roots, (2) whether the diversity and richness of fungal community in the continuous cropping of soybean roots and rhizosphere soil would be changed after inoculation, (3) whether F. mosseae could affect the community composition of rhizosphere fungi in soybean under different continuous cropping regimes. The results will provide theoretical and technical guidance for biological methods to alleviate the obstacles of soybean under continuous cropping.

\section{MATERIALS AND METHODS}

\section{Soybean cultivar and mycorrhiza inocula}

The soybean cultivar was Suinong 26 in the present study (38.80\% average protein and $21.59 \%$ average fat content). The soybean cultivar was purchased from Heilongjiang Academy of Agricultural Sciences, Harbin, China.

The AM fungus for inoculation was Funneliformis mosseae which was obtained from the rhizosphere soil of soybean in Heilongjiang Province of China. The F. mosseae inocula were propagated with clover using vermiculite and river sand as the substrate. The F. mosseae inocula consisted of root segments, hyphae, spores and substrate with about 20-30 spores per gram.

\section{Experimental design}

This study was carried out at the Experimental Station of the Research Institute of Sugar Industry, Harbin Institute of

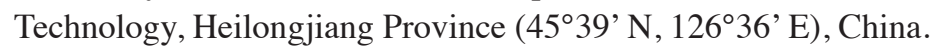

Soybean seeds were superficially disinfected in ethanol (70\%) for $1 \mathrm{~min}$, then in sodium hypochlorite (25\%) for $5 \mathrm{~min}$, and finally rinsed with sterile distilled water for at least 10 times. The disinfected soybean seeds were placed in petri dishes with sterile filter paper and moist cotton and were kept in the dark at $28{ }^{\circ} \mathrm{C}$ until the soybean radicle reached about $2 \mathrm{~cm}$.

Potted plants were used for our study, with $4 \mathrm{~kg}$ air-dried and sieved through a $4 \mathrm{~mm}$ mesh soil in each pot. Soils were labeled as experimental groups and control groups with 0,1 , and $3 \mathrm{yr}$ of continuous cropping for soybean, respectively. The experimental group soils were inoculated with $45 \mathrm{~g}$ F. mosseae inocula in each pot and mixed well, while the control group soils were non-inoculated with $F$. mosseae inocula. No other fertilizers were applied throughout the experiment. The soybean seedlings were sown in pots (three seedlings per pot) containing the above soils in May 2017. Each treatment was composed by nine replicates. All the plants were grown in the greenhouse under controlled conditions: temperature of $23 \pm 1{ }^{\circ} \mathrm{C}$, photoperiod $12: 12 \mathrm{~h}$ and humidity $>60 \%$. The pots were watered daily. The whole experiment was repeated three times. The samples were randomly collected at the seedling stage ( $30 \mathrm{~d}$ after seedling emergence) of the soybean cv. SN26. Samples from three pots were randomly selected for each treatment. The soils were collected at 0-20 depth to form samples. Some of the roots and soil samples were stored at $-80^{\circ} \mathrm{C}$ before DNA extraction, while the other root samples were used for other analyses. 


\section{Determination of disease index of soybean root rot and community composition of rhizosphere fungi}

Soybean roots were selected randomly from each treatment. A root rot rating was described according to Zhou et al. (2011). In order to reduce the random error, three experiments were carried out in parallel, and average values determined for each group of parallel experiments.

Rhizosphere soil DNA was extracted with PowerSoil DNA isolation kit (MO BIO Laboratories, Carlsbad, California, USA). Roots DNA was extracted according to Long et al. (2005). ITS1F (CTTGGTCATTTAGAGGAAGTAA) and ITS2 (GCTGCGTTCTTCATCGATGC) primers were used to amplify the internal transcribed spacer 1 (ITS1) region (Smith and Peay, 2014). PCR reactions were performed in a $50 \mu \mathrm{L}$ reaction containing $5 \times$ FastPfu buffer $10 \mu \mathrm{L}, 2.5 \mathrm{mM}$ dNTPs $5 \mu \mathrm{L}, 5 \mu \mathrm{M}$ ITS1F primer $2.0 \mu \mathrm{L}, 5 \mu \mathrm{M}$ ITS2 primer $2.0 \mu \mathrm{L}, 1.0 \mathrm{U}$ Taq polymerase $0.2 \mu \mathrm{L}$, template DNA $5 \mu \mathrm{L}$, and finally sterile $\mathrm{ddH}_{2} \mathrm{O}$ was added to $50 \mu \mathrm{L}$. PCR conditions were as follows: $98{ }^{\circ} \mathrm{C}$ for $2 \mathrm{~min}, 25$ cycles of $98^{\circ} \mathrm{C}$ for $30 \mathrm{~s}, 50^{\circ} \mathrm{C}$ for $30 \mathrm{~s}$ and $72^{\circ} \mathrm{C}$ for $1 \mathrm{~min}$, followed by $72{ }^{\circ} \mathrm{C}$ for $5 \mathrm{~min}$. Amplicons were purified using a GeneJET Gel Extraction Kit (Thermo Scientific, Waltham, Massachusetts, USA) and quantified using a Qubit dsDNA HS Assay Kit (Life Technologies, Carlsbad, California, USA).

The comparisons of the fungal community were analyzed by high-throughput pyrosequencing technology (Illumina HiSeq 2500, BioMarker Technologies, Beijing, China) to determine how the diversity and richness of fungal community changed in response to different continuous cropping regimes and $F$. mosseae. The raw paired-end reads were joined with FLASH v1.2.7 (Magoc and Salzberg, 2011). The raw reads were processed using QIIME (V1.8.0) (Caporaso et al., 2010). The high-quality reads were clustered through the UCLUST (version 1.2.22) (Edgar et al., 2011) at a 0.97 sequence similarity level to generate different operational taxonomic units (OTUs) (Bokulich et al., 2013). The richness indexes Ace (Hughes et al., 2001), Chao1 (Chao et al., 2005), Simpson (Simpson, 1949), and Shannon (Rodrigues et al., 2014), and Good's Coverage (Rodrigues et al., 2014) were evaluated. The community structure was analyzed and compared. After clustering, a heatmap was calculated to show the relative differences in OTU abundances in the samples (Bai et al., 2015). The raw sequences were deposited into the National Center for Biotechnology Information (NCBI) Sequence Read Archive (SAR) database (accession number SRP161450).

\section{Statistical analysis}

An ANOVA was conducted by SPSS 19.0 statistical software (SPSS Inc., Chicago, Illinois, USA). Two-way ANOVA was used to compare the interactive effects of the inoculation of F. mosseae, continuous cropping regimes and their interactions on the disease index of soybean root rot. A Tukey's test (honestly significant differences, HSD) was performed to determine the significant differences $(\mathrm{P}<0.05)$ between means.

\section{RESULTS}

Effect of $\boldsymbol{F}$. mosseae on disease index of soybean root rot under continuous cropping regimes

Morphological observation was used to analyze the effect of $F$. mosseae on the disease index of soybean root rot under different continuous cropping regimes during seedling period. The root rot disease index was significantly affected by the inoculation of F. mosseae compared with non-inoculation seedlings under the same continuous cropping regimes (Table 1). The root rot disease index decreased significantly for all the plants inoculated with F. mosseae compared with control. For example, the root rot disease index was 0.73 -fold for sample in0Y in comparison with the values of sample non0Y (Table 1). Moreover, we found that the root rot disease index was significantly affected by the increasing of continuous cropping regimes. Significant differences were found in root rot disease index under different continuous cropping regimes. From Table 1, we clearly found that the root rot index in zero year of continuous cropping was the lowest, which was only

Table 1. Detection of the disease index of soybean root rot.

\begin{tabular}{ccccccc}
\hline & non0Y & in0Y & non1Y & in1Y & non3Y & in3Y \\
\hline Root rot disease index & $0.81 \pm 0.01 \mathrm{~d}$ & $0.59 \pm 0.01 \mathrm{e}$ & $1.16 \pm 0.03 \mathrm{~b}$ & $0.82 \pm 0.02 \mathrm{~d}$ & $1.27 \pm 0.03 \mathrm{a}$ & $0.94 \pm 0.01 \mathrm{c}$ \\
\hline
\end{tabular}

Non: non-inoculated with Funneliformis mosseae; in: inoculated with F. mosseae

$0 \mathrm{Y}, 1 \mathrm{Y}$ and $3 \mathrm{Y}$ represent $0 \mathrm{yr}, 1 \mathrm{yr}$ and $3 \mathrm{yr}$ of continuous cropping, respectively.

Different letters indicate significant differences from different treatments $(\mathrm{P}<0.05)$. 
0.81 , followed by $1 \mathrm{yr}$ of continuous cropping (1.16), and finally $3 \mathrm{yr}$ of continuous cropping (1.27). The value of root rot disease index had nonsignificant differences between non-inoculated seedlings under zero year of continuous cropping and inoculated seedlings under $1 \mathrm{yr}$ of continuous cropping. Two-way ANOVA results showed that the interactive effects of the inoculation of F. mosseae and continuous cropping regimes were significantly affected the disease index of soybean root rot (Table 2). Overall, the results clearly indicated that the inoculation of $F$. mosseae significantly decreased the disease index of soybean root rot under different continuous cropping regimes.

\section{Overall HiSeq sequencing information and fungal community}

In total, 845596 valid sequences from the 12 samples were obtained; 801015 optimized sequences were finally retained after filtering. When grouped at the 0.97 sequence similarity, there were 725 OTUs for all of the samples. 58017 sequences of each sample were randomly selected for following data analyses, and the $696204(58017 \times 12)$ sequences were clustered into 725 OTUs. The Alpha-diversity of the OTUs in the 12 samples was shown in Table 3, and the coverage of these 12 libraries was all above 99.9\%. The smaller the Simpson index or the larger the Shannon index, the lower the community diversity. The mean values of OTU richness, the Ace, Chao1 and Shannon indexes of the six soil samples were higher than those of the six root samples under the same experimental treatment, while the opposite results occurred for the Simpson index (Table 3). Furthermore, the mean values of OTU richness, the Ace, Chao1 and Shannon indexes were all highest in non0YSF, but the Simpson index was the lowest in non0YSF. It also indicated that the fungal community diversity of all the root samples increased with the increase of continuous cropping regimes.

\section{Comparisons of fungal community}

The relative abundance of fungal community composition of the 12 samples at the phylum level is shown in Figure 1(a). With regard to the composition proportions, Ascomycota was the dominant phylum in the samples except non1YRF, which with a dominance phylum of Olpidiomycota. Moreover, the sequences of Ascomycota occupied more than $43.58 \%$ of the total amount in all the soil samples. Basidiomycota was the second dominant phylum in all the soil samples, but Olpidiomycota was the second phylum in the roots except in0YRF. The Mortierellomycota population in all the soil

Table 2. Two-way ANOVA for inoculation with Funneliformis mosseae (I), continuous cropping regimes (Y) and their interactions on the disease index of soybean root rot.

\begin{tabular}{lcccc}
\hline Index & Item & $\begin{array}{c}\text { Inoculation with } \\
\text { F. mosseae }(\mathrm{I})\end{array}$ & $\begin{array}{c}\text { Continuous cropping } \\
\text { regimes }(\mathrm{Y})\end{array}$ & $\mathrm{I} \times \mathrm{Y}$ \\
\hline Root rot disease index & $\mathrm{df}$ & 1 & 2 & 2 \\
& $\mathrm{MS}$ & 0.396 & 0.268 & 0.007 \\
& F-ratio & $308.610^{* *}$ & $209.121^{* *}$ & $5.182^{*}$ \\
\hline
\end{tabular}

*Factors are significant at $\mathrm{P}<0.05$ level.

$* *$ Factors are significant at $\mathrm{P}<0.01$ level.

Table 3. Diversity indices of fungal community in the twelve samples.

\begin{tabular}{lcccccc}
\hline Sample ID & OTU & Ace & Chao1 & Simpson & Shannon & Coverage \\
\hline in0YRF & 313 & 387.2412 & 414.5278 & 0.1986 & 2.4148 & 0.9985 \\
in0YSF & 559 & 564.0678 & 568.5625 & 0.0330 & 4.5119 & 0.9997 \\
in1YRF & 228 & 287.9191 & 283.4545 & 0.3066 & 1.9522 & 0.9990 \\
in1YSF & 576 & 585.2117 & 589.0435 & 0.0310 & 4.5491 & 0.9996 \\
in3YRF & 202 & 276.0381 & 265.1714 & 0.4498 & 1.4382 & 0.9990 \\
in3YSF & 555 & 564.2016 & 569.8824 & 0.0477 & 4.4808 & 0.9996 \\
non0YRF & 402 & 442.0222 & 449.6667 & 0.1550 & 2.5491 & 0.9989 \\
non0YSF & 603 & 613.2606 & 623.3125 & 0.0177 & 4.9981 & 0.9996 \\
non1YRF & 285 & 333.5670 & 337.3171 & 0.1897 & 2.3452 & 0.9990 \\
non1YSF & 571 & 580.0186 & 581.5238 & 0.0586 & 4.0450 & 0.9993 \\
non3YRF & 205 & 244.8282 & 236.8750 & 0.5209 & 1.2750 & 0.9992 \\
non3YSF & 539 & 554.0316 & 567.9565 & 0.0477 & 4.1160 & 0.9994 \\
\hline
\end{tabular}

In: Inoculated with Funneliformis mosseae; non: non-inoculated with F. mosseae; RF: fungi in root samples; SF: fungi in soil samples.

$0 \mathrm{Y}, 1 \mathrm{Y}$ and $3 \mathrm{Y}$ represent $0 \mathrm{yr}, 1 \mathrm{yr}$ and $3 \mathrm{yr}$ of continuous cropping, respectively. 
Figure 1. Relative abundances of the different fungal communities at the phylum level (a) and the genus level (b) in the 12 samples.
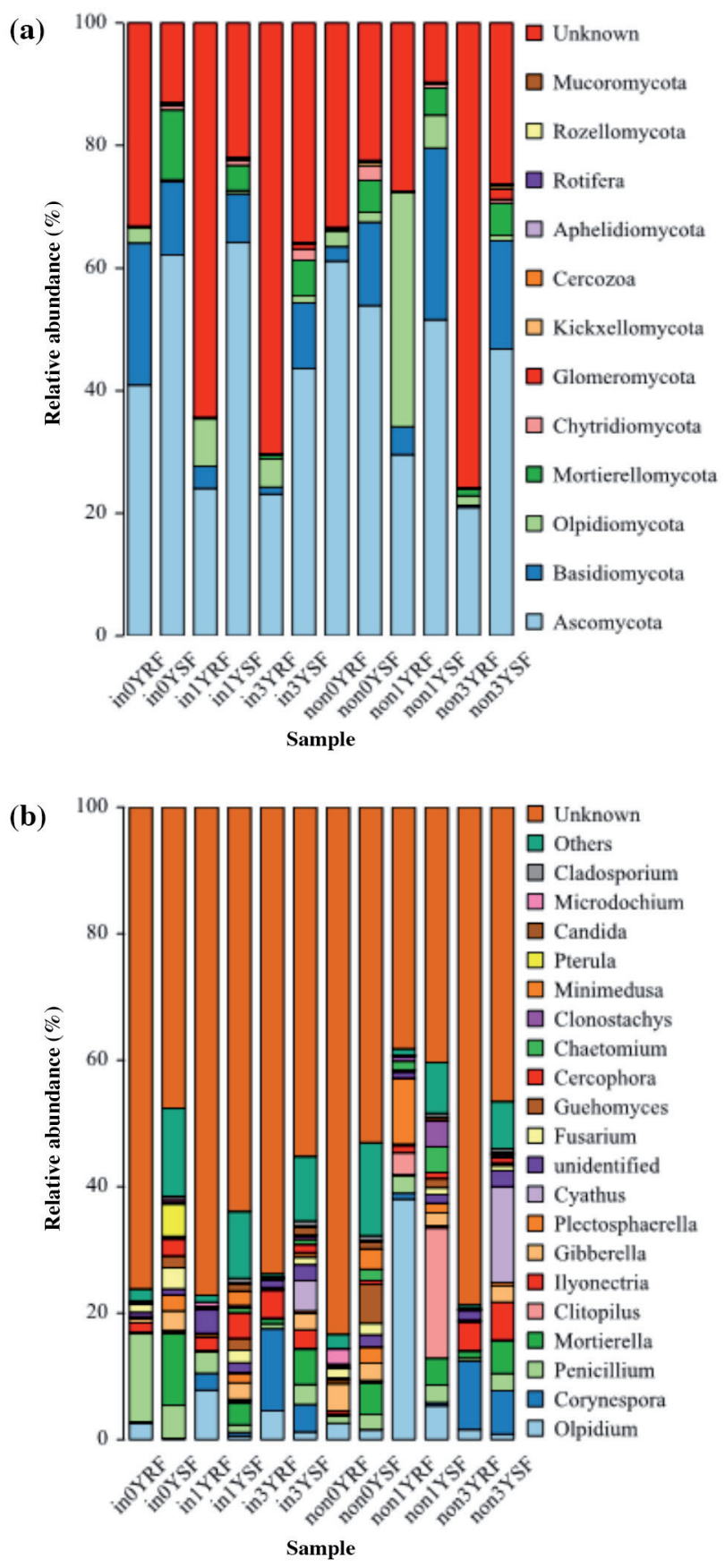

In: Inoculated with F. mosseae; non: non-inoculated with Funneliformis mosseae; RF: fungi in root samples; SF: fungi in soil samples. $0 \mathrm{Y}, 1 \mathrm{Y}$ and $3 \mathrm{Y}$ represent $0 \mathrm{yr}, 1 \mathrm{yr}$ and $3 \mathrm{yr}$ of continuous cropping, respectively.

samples was significantly higher than that in the roots. An important phylum (Glomeromycota) was observed in all the samples. In addition, other important phyla (Chytridiomycota, Kickxellomycota, Aphelidiomycota, and Mucoromycota) were observed in all the soil samples. However, there were significant differences in the 12 identified phyla between inoculation and non-inoculation of F. mosseae samples.

At the genus level, Penicillium content in in0YRF (13.95\%) was much higher than the other samples (Figure 1(b)). Plant pathogenic fungi, such as Olpidium (2.52\%), Ilyonectria (1.46\%), Fusarium (1.32\%), Gibberella (0.66\%), 
Plectosphaerella (0.34\%), Corynespora (0.28\%), Microdochium (0.21\%), and Cladosporium (0.02\%), were also detected in in0YRF. However, Penicillium content decreased significantly and kept a low level about $1.15 \%$ in non0YRF. The predominant identified pathogens were Gibberella (4.23\%), Olpidium (2.51\%), Microdochium (2.39\%), Fusarium $(1.45 \%)$, Plectosphaerella $(0.60 \%)$, Ilyonectria $(0.53 \%)$, Cladosporium $(0.10 \%)$, and Corynespora $(0.09 \%)$ in non0YRF. The genus level analysis demonstrated that the inoculation of F. mosseae could favor Penicillium over Gibberella and Olpidium in in0YRF. As was shown in Figure 1b, non1YRF was different from other roots non-inoculated with $F$. mosseae. Olpidium content maintained a high level about 38.04\% in non1YRF. In addition, in1YRF was also different from in0YRF and in 3YRF. The dominant identified genus was also Olpidium (7.78\%), followed by Penicillium (3.39\%) and Corynespora (2.68\%) in in1 YRF. In in3YRF and non3YRF, Corynespora was the most prevalent genus, followed by Ilyonectria and Olpidium. Moreover, the relative abundance of Fusarium in the three inoculated root samples was lower than those in the three non-inoculated root samples in the same continuous cropping regime. In light of this, both inoculation and continuous cropping regimes had significant effects on the dominant genus and their relative abundances in the roots. Compared with roots, lower amount of Olpidium and Corynespora were observed in the soil samples. However, Mortierella and Cercophora content increased significantly and maintained a high level at least 3.52\% and $0.61 \%$ in the soil samples, respectively. Comparison of the genus-level proportional abundances showed that the major genera among the soil samples were different, and their relative abundances were also different. For the soil samples, the predominant identified fungi were Mortierella and Gibberella. It indicated that F. mosseae might have significant effects on the composition of rhizospheric fungal community, which was similar to those observed at the phylum level.

Clustering results for the dominant fungal communities at the genus level in the 12 samples were showed on a heatmap (Figure 2). According to the similarities among the compositions, all the root samples were divided into two groups, one for non0YRF and the remaining samples clustered together. It was consistent with the relative abundances of the different fungal communities at the genus level. As shown in Figure 2, all the soil samples were divided into the following three groups: non1YSF, in1YSF and in3YSF clustered together; non0YSF and in0YSF clustered together, which indicated a similar community structure between the two soil samples; non3YSF did not cluster with other soil samples. The results showed that both the continuous cropping regimes and inoculation of $F$. mosseae could significantly affect the dominant genera and their relative abundances in the roots and soil samples.

\section{DISCUSSION}

A variety of AM fungi can coexist in agricultural ecosystems. However, even if there are a large number of native AM fungal spores in natural soil, it is usually composed by a poor community with functionally redundant species, which may result in a decrease in the number of functions performed by AM fungi (Mendes et al., 2015). Moreover, competition may exist between native AM fungi and nonnative AM fungi (Buysens et al., 2017). The effects of native AM fungi on plant growth were more effective than that of nonnative AM fungi, which may be due to the adaptation to soil factors (Diagne et al., 2018). In our study, the AM fungus for inoculation was obtained from the rhizosphere soil of soybean in Heilongjiang Province of China. The native AM inoculum was more effective than nonnative AM fungi, allowing coexistence with other native AM in soybean continuous cropping soil. In natural soil where native AM fungi may already have formed a complete network of hyphae, additional native AM fungi may be more likely to become dominant AM fungi. The introduced AM fungi became dominant, indicating that the community of the native AM fungi in the roots of host plants had changed greatly (Koch et al., 2011; Garg and Rekha, 2015). The introduced AM fungi can also directly interact with the resident genotypes of the same species, because they are closely related, but genetically distinct AM fungi can coincide with each other and exchange genetic information (Croll and Sanders, 2009). Furthermore, in soybean continuous cropping soil where a community of the native AM fungi was already present, inoculation by $F$. mosseae decreased the root rot incidence of soybean roots (Table 1). It suggests that there may be functional and physiological complementarity among these AM fungi (Eun-Hwa et al., 2013). Native AM fungi are also important contributors to ecosystem productivity (Caravaca et al., 2005). The persistence and abundance of additional native AM fungus could be promoted by the presence of other native AM fungi, even if the additional native AM fungus was not the most efficient one (Thioye et al., 2019). The potential of native AM fungi and F. mosseae in improving plant disease resistance makes it a promising biological tool, which can be used to alleviate the obstacles of soybean continuous cropping. 
Figure 2. Heatmap analysis of similarities among the dominant fungal communities at the genus level in the 12 samples. The relative abundances of fungal genera are described by color intensity.

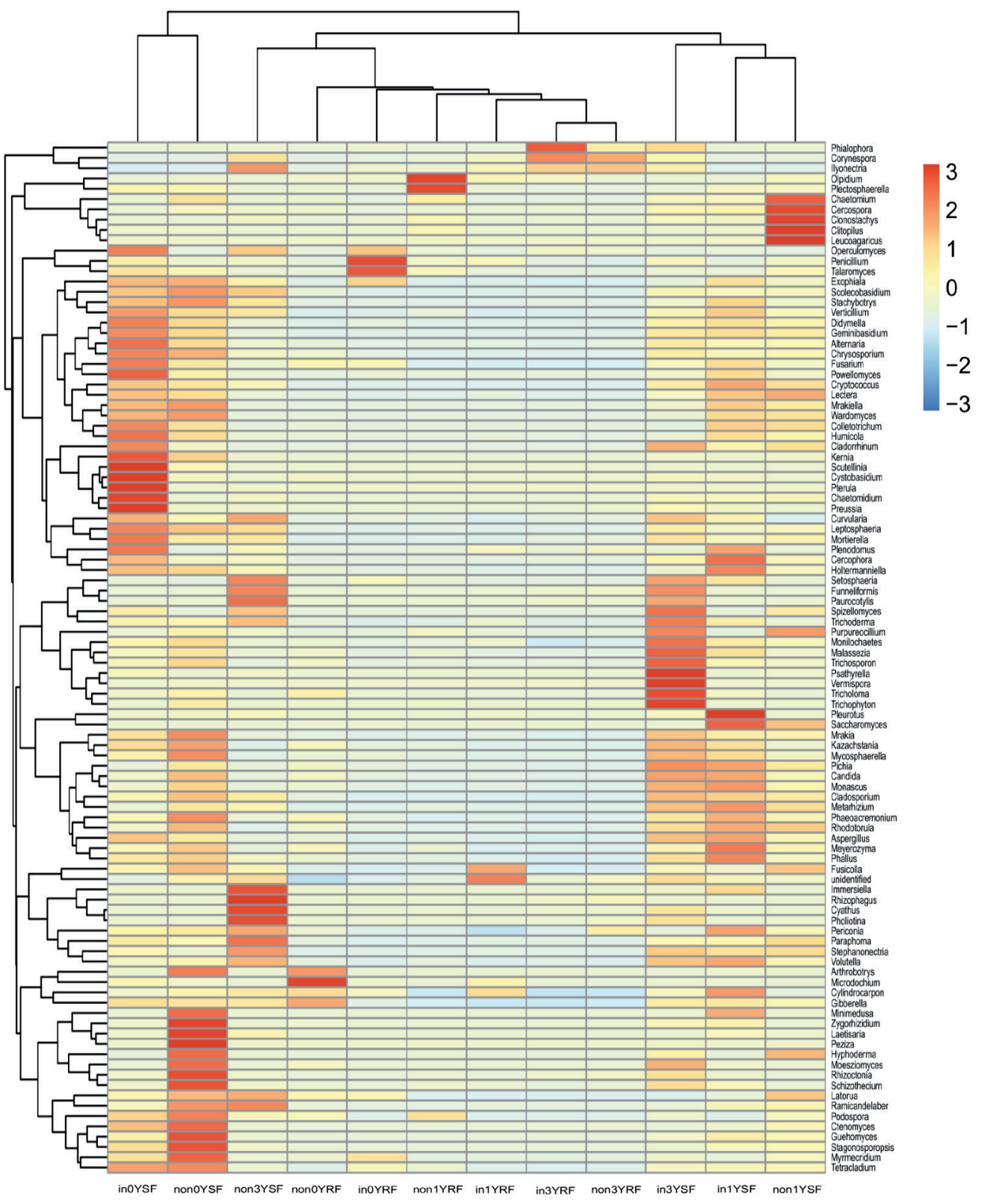

In: Inoculated with Funneliformis mosseae; non: non-inoculated with F. mosseae; RF: fungi in root samples; SF: fungi in soil samples. $0 \mathrm{Y}, 1 \mathrm{Y}$ and $3 \mathrm{Y}$ represent $0 \mathrm{yr}, 1 \mathrm{yr}$ and $3 \mathrm{yr}$ of continuous cropping, respectively.

In our study, we found that the disease index of soybean root rot was significantly higher in control non-inoculated with $F$. mosseae than that in the treatment, indicating that inoculation of $F$. mosseae could significantly improve the resistance of soybean to soil-borne pathogens, ultimately alleviated the occurrence of soybean root rot. Previous studies have indicated that AM fungi could significantly inhibit some soil-borne pathogens, such as species of Phytophthora, Rhizoctonia and Fusarium (Abdel-Fattah et al., 2011; Sukhada et al., 2011; Eke et al., 2016). In our previous study, we have shown that species of Fusarium are the main parasitic pathogens of soybean root rot in Northeast China. Intriguingly, our results showed that the relative abundance of Fusarium in all the root samples inoculated with F. mosseae was lower than those in the three non-inoculated root samples (Figure 1b). The results demonstrated that the symbiosis of AM fungi 
could improve the resistance of soybean plants to root rot. The priming of plant defense responses enhances the basic resistance of plants, making it possible for plants to respond quickly to the attacks of pathogenic microorganisms (Ahmad et al., 2010). Considering their unique advantages in biological control potential, AM fungi should provide new ways to protect crops from the attacks of soil-borne pathogenic microorganisms in sustainable agriculture.

In addition, the interaction between AM fungi and their host plants plays an important role in the formation of rhizosphere microbial communities (Cameron et al., 2013). Based on the phylum and genus level analysis (Figure 1), we found that the fungal abundance distribution in all the samples was affected by inoculation of $F$. mosseae. The variation of fungal abundance in our study may be related to the indirect changes in the soil environmental factors caused by F. mosseae. AM fungi can improve the biosynthesis of some beneficial phytochemicals and increase the activity of antioxidant enzymes (Schweiger and Müller, 2015). It improves micro-environments and provides substrates for microbial growth, eventually leading to changes in soil fungal abundance. Meanwhile, comparison of the phylum and genus level proportional abundances showed that the major phyla and genera among the samples were different, and their relative abundances were also different. Continuous cropping converts neutral soil into acidic soil, which benefits the growth of fungi, but inhibits the growth of bacteria and actinomycetes. Eventually, continuous cropping of soybean leads to significant changes of the microbial communities in the rhizosphere soil. In this study, the introduction of $F$. mosseae to the system of soybean continuous cropping changed the rhizosphere fungal community as a whole. Further and long-term studies are needed using changes of multiple microbial communities to confirm that $F$. mosseae and continuous cropping affect rhizosphere microorganisms. Additionally, further understanding of the microbial communities in the rhizosphere soil under different continuous cropping regimes may give insight into mechanisms behind continuous cropping obstacles and help determine optimal practices for maintaining productive soil.

The community composition of rhizosphere fungi in soybean continuous cropping soil was analyzed using highthroughput sequencing in this study. This provided the necessary conditions for further research on the fungal community composition in soybean continuous cropping soil, and the analysis of fungal community composition indicated the dominant phyla and genera. As shown in Figure 1, many unknown and unidentified sequences/phyla/genera were detected. At the genus level, the top genera, accounting for at least $38.18 \%$ of the total abundance were unknown genera in the 12 samples. The results indicated that there were many unknown microorganisms in the rhizosphere fungal communities of continuous cropping soybean, which laid a foundation for further study on continuous cropping obstacles. Moreover, the unidentified genera accounted for only $0.31 \%$ of the total community in the samples. Detection of unknown and unidentified members of the microbial communities in our study shows that high-throughput sequencing is highly efficient in detecting microorganisms, especially uncultured and rare species. As far as we know, this is the first direct evidence that the community composition of rhizosphere fungi in soybean continuous cropping soil is changed by $F$. mosseae based on a high-throughput sequencing method. The results of this study would help to alleviate the obstacles of soybean continuous cropping by biological approaches.

\section{CONCLUSIONS}

In this study, the effects of Funneliformis mosseae on disease index of soybean root rot and the community composition of rhizosphere fungi in soybean continuous cropping soils were studied during the seedling period. It clearly demonstrated that inoculation of $F$. mosseae significantly decreased the disease index of soybean root rot and changed the community composition of rhizosphere fungi in the continuous cropping of soybean during the seedling period. The root rot disease index was also affected by the increasing of continuous cropping regimes. The results can provide new insights into the interactive effects of AM fungi and rhizosphere fungi, and also provide theoretical evidence on biological solutions to alleviate the obstacles of soybean continuous cropping.

\section{ACKNOWLEDGEMENTS}

This work was supported by a grant from Natural Science Foundation of China (31570487), Key project of horizontal subject of Heilongjiang East University (HDFHX160103, HDFHX160111), Open Foundation of Key Laboratory of Saline-alkali Vegetation Ecology Restoration in Oil Field, Ministry of Education (SAVER 1611). Weiguang Jie and Jixiang Lin contributed equally to this work. 


\section{REFERENCES}

Abdel-Fattah, G.M., El-Haddad, S.A., Hafez, E.E., and Rashad, Y.M. 2011. Induction of defense responses in common bean plants by arbuscular mycorrhizal fungi. Microbiological Research 166:268-281.

Ahmad, S., Gordon-Weeks, R., Pickett, J., and Ton, J. 2010. Natural variation in priming of basal resistance: from evolutionary origin to agricultural exploitation. Molecular Plant Pathology 11:817-827.

Bai, L., Cui, J.Q., Jie, W.G., and Cai, B.Y. 2015. Analysis of the community compositions of rhizosphere fungi in soybeans continuous cropping fields. Microbiological Research 180:49-56.

Battini, F., Turrini, A., Quartacci, M.F., Malorgio, F., Sgherri, C., Picciarelli, P., et al. 2016. Dual inoculation with AMF and associated bacteria improves nutraceutical value of sweet basil grown under commercial conditions. Agrochimica 60:81-99.

Berg, G., and Smalla, K. 2009.Plant species and soil type cooperatively shape the structure and function of microbial communities in the rhizosphere. FEMS Microbiology Ecology 68:1-13.

Bokulich, N.A., Subramanian, S., Faith, J.J., Gevers, D., Gordon, J.I., Knight, R., et al. 2013. Quality-filtering vastly improves diversity estimates from Illumina amplicon sequencing. Nature Methods 10:57-59.

Buysens, C., Alaux, P.L., César, V., Huret, S., Declerck, S., and Cranenbrouck, S. 2017. Tracing native and inoculated Rhizophagus irregularis in three potato cultivars (Charlotte, Nicola and Bintje) grown under field conditions. Applied Soil Ecology 115:1-9.

Cameron, D.D., Neal, A.L., van Wees, S.C.M., and Ton, J. 2013. Mycorrhiza-induced resistance: more than the sum of its parts? Trends in Plant Science 18:539-545.

Caporaso, J.G., Kuczynski, J., Stombaugh, J., Bittinger, K., Bushman, F.D., Costello, E.K., et al. 2010. QIIME allows analysis of high-throughput community sequencing data. Nature Methods 7:335-336.

Caravaca, F., Alguacil, M.M., Barea, J.M., and Roldán, A. 2005. Survival of inocula and native AM fungi species associated with shrubs in a degraded Mediterranean ecosystem. Soil Biology and Biochemistry 37:227-233.

Chao, A., Chazdon, R.L., Colwell, R.K., and Shen, T.J. 2005. A new statistical approach for assessing compositional similarity based on incidence and abundance data. Ecology Letters 8:148-159.

Costa, R., Götz, M., Mrotzek, N., Lottmann, J., Berg, G., and Smalla, K. 2006. Effects of site and plant species on rhizosphere community structure as revealed by molecular analysis of microbial guilds. FEMS Microbiology Ecology 56:236-249.

Croll, D., and Sanders, I.R. 2009. Recombination in Glomus intraradices, a supposed ancient asexual arbuscular mycorrhizal fungus. BMC Evolutionary Biology 9:13.

Deng, S.Q., Ke, T., Li, L.T., Cai, S.W., Zhou, Y.Y., Liu, Y., et al. 2018. Impacts of environmental factors on the whole microbial communities in the rhizosphere of a metal-tolerant plant: Elsholtzia haichowensis Sun. Environmental Pollution 237:1088-1097.

Dhawi, F., Datta, R., and Ramakrishna, W. 2015. Mycorrhiza and PGPB modulate maize biomass, nutrient uptake and metabolic pathways in maize grown in mining-impacted soil. Plant Physiology and Biochemistry 97:390-399.

Diagne, N., Baudoin, E., Svistoonoff, S., Ouattara, C., Diouf, D., Kane, A., et al. 2018. Effect of native and allochthonous arbuscular mycorrhizal fungi on Casuarina equisetifolia growth and its root bacterial community. Arid Land Research and Management 32:212-228.

Edgar, R.C., Haas, B.J., Clemente, J.C., Quince, C., and Knight, R. 2011. UCHIME improves sensitivity and speed of chimera detection. Bioinformatics 27:2194-2200.

Eke, P., Chatue, G.C., Wakam, L.N., Kouipou, R.M.T., Fokou, P.V.T., and Boyom, F.F. 2016. Mycorrhiza consortia suppress the fusarium root rot (Fusarium solani f. sp. Phaseoli) in common bean (Phaseolus vulgaris L.). Biological Control 103:240-250.

Eun-Hwa, L., Ju-Kyeong, E., Kang-Hyeon, K., and Ahn-Heum, E. 2013. Diversity of arbuscular mycorrhizal fungi and their roles in ecosystems. Mycobiology 41:121-125.

Garbeva, P., van Veen, J.A., and van Elsas, J.D. 2004. Microbial diversity in soil: selection of microbial populations by plant and soil type and implications for disease suppressiveness. Annual Review of Phytopathology 42:243-270.

Garg, N., and Rekha, P. 2015. Effectiveness of native and exotic arbuscular mycorrhizal fungi on nutrient uptake and ion homeostasis in salt-stressed Cajanus cajan L. (Millsp.) genotypes. Mycorrhiza 25:165-180.

Gawade, D.B., Perane, R.R., Suryawanshi, A.P., and Deokar, C.D. 2017. Extracellular enzymes activity determining the virulence of Rhizoctonia bataticola, causing root rot in soybean. Physiological and Molecular Plant Pathology 100:49-56.

Huang, L.F., Song, L.X., Xia, X.J., Mao, W.H., Shi, K., Zhou, Y.H., et al. 2013. Plant-soil feedbacks and soil sickness: from mechanisms to application in agriculture. Journal of Chemical Ecology 39:232-242.

Hughes, J.B., Hellmann, J.J., Ricketts, T.H., and Bohannan, J.M. 2001. Counting the uncountable: statistical approaches to estimating microbial diversity. Applied and Environmental Microbiology 67:4399-4406.

Jeon, C.S., Kim, G.H., and Koh, Y.J. 2013. Root rot of balloon flower (Platycodon grandiflorum) caused by Fusarium solani and Fusarium oxysporum. Plant Pathology Journal 29:440-445.

Koch, A.M., Antunes, P.M., Barto, E.K., Cipollini, D., Mummey, D.L., and Klironomos, J.N. 2011. The effects of arbuscular mycorrhizal (AM) fungal and garlic mustard introductions on native AM fungal diversity. Biological Invasions 13:1627-1639. 
Kong, A.Y.Y., Scow, K.M., Córdova-Kreylos, A.L., Holmes, W.E., and Six, J. 2011. Microbial community composition and carbon cycling within soil microenvironments of conventional low-input, and organic cropping systems. Soil Biology and Biochemistry 43:20-30.

Li, J.G., Ren, G.D., Jia, Z.J., and Dong, Y.H. 2014. Composition and activity of rhizosphere microbial communities associated with healthy and diseased greenhouse tomatoes. Plant and Soil 380:337-347.

Liu, J.J., Yu, Z.H., Yao, Q., Hu, X.J., Zhang, W., Mi, G., et al. 2017. Distinct soil bacterial communities in response to the cropping system in a Mollisol of northeast China. Applied Soil Ecology 119:407-416.

Long, L.K., Yang, S.Z., Yao, Q., and Zhu, H.H. 2005. DNA extraction from arbuscular mycorrhizal fungi and analysis by PCRdenaturing gradient gel electrophoresis. Mycosystema 24:564-569. [In Chinese].

Luan, F.G., Zhang, L.L., Lou, Y.Y., Wang, L., Liu, Y.N., and Zhang, H.Y. 2015. Analysis of microbial diversity and niche in rhizosphere soil of healthy and diseased cotton at the flowering stage in southern Xinjiang. Genetics and Molecular Research 14:1602-1611.

Magoc, T., and Salzberg, S.L. 2011.FLASH: fast length adjustment of short reads to improve genome assemblies. Bioinformatics 27:2957-2963

Mendes, L.W., Tsai, S.M., Navarrete, A.A., De Hollander, M., van Veen, J.A., and Kuramae, E.E. 2015. Soil-borne microbiome: linking diversity to function. Microbial Ecology 70:255-265.

Rodrigues, V.D., Torres, T.T., and Ottoboni, L.M.M. 2014. Bacterial diversity assessment in soil of an active Brazilian copper mine using high-throughput sequencing of 16S rDNA amplicons. Antonie Van Leeuwenhoek 106:879-890.

Schweiger, R., and Müller, C. 2015. Leaf metabolome in arbuscular mycorrhizal symbiosis. Current Opinion Plant Biology 26:120-126.

Simpson, E.H. 1949. Measurement of diversity. Nature 163:688.

Smith, D.P., and Peay, K.G. 2014. Sequence depth, not PCR replication, improves ecological inference from next generation DNA sequencing. PLOS ONE 9:e90234.

Spagnoletti, F., Carmona, M., Gómez, N.E.T., Chiocchio, V., and Lavado, R.S. 2017. Arbuscular mycorrhiza reduces the negative effects of M.phaseolina on soybean plants in arsenic-contaminated soils. Applied Soil Ecology 121:41-47.

Spatafora, J.W., Chang, Y., and Benny, G.L. 2016. A phylum-level phylogenetic classification of zygomycete fungi based on genome-scale data. Mycologia 108:1028-1046.

Sugiyama, A., Ueda, Y., Zushi, T., Takase, H., and Yazaki, K. 2014. Changes in the bacterial community of soybean rhizospheres during growth in the field. PLOS ONE 9:e100709.

Sukhada, M., Manjula, R., and Rawal, R.D. 2011. Evaluation of arbuscular mycorrhiza and other biocontrol agents against Phytophthora parasitica var. nicotianae infecting papaya (Carica papaya cv. Surya) and enumeration of pathogen population using immunotechniques. Biological Control 58:22-29.

Thioye, B., Sanguin, H., Kane, A., Faria, S.M., Fall, D., Prin,Y., et al. 2019. Impact of mycorrhiza-based inoculation strategies on Ziziphus mauritiana Lam. and its native mycorrhizal communities on the route of the Great Green Wall (Senegal). Ecological Engineering 128:66-76.

Wang, Y.Y., Yin, Q.S., Qu, Y., Li, G.Z., and Hao, L. 2018. Arbuscular mycorrhiza-mediated resistance in tomato against Cladosporium fulvum-induced mould disease. Journal of Phytopathology 166:67-74.

Wright, S.F., and Upadhyaya, A. 1998. A survey of soils for aggregate stability and glomalin, a glycoprotein produced by hyphae of arbuscular mycorrhizal fungi. Plant and Soil 198:97-107.

Wu, Z.X., Hao, Z.P., Sun, Y.Q., Guo, L.P., Huang, L.Q., Zeng, Y., et al. 2016. Comparison on the structure and function of the rhizosphere microbial community between healthy and root-rot Panax notoginseng. Applied Soil Ecology 107:99-107.

Zhou, N., Liu, P., and Xu, G.D. 2011. The effects of rapeseed root exudates on the forms of aluminum in aluminum stressed rhizosphere soil. Crop Protection 30:631-636.

Zhu, Y., Lv, G.C., Chen, Y.L., Gong, X.F., Peng, Y.N., Wang, Z.Y., et al. 2017. Inoculation of arbuscular mycorrhizal fungi with plastic mulching in rainfed wheat: A promising farming strategy. Field Crops Research 204:229-241. 